Cáceres, C., Muñoz, C. \& Valenzuela, J. (2021). Responsabilidad personal docente y motivación escolar. Revista Electrónica Interuniversitaria de Formación del Profesorado, 24(1), 175-188.

DOI: https://doi.org/10.6018/reifop.402761

\title{
Responsabilidad personal docente y motivación escolar
}

\author{
Consuelo Cáceres ${ }^{1}$, Carla Muñoz ${ }^{2}$ y Jorge Valenzuela \\ 'Escuela de Pedagogía, Pontificia Universidad Católica de Valparaíso \\ ${ }^{2}$ Departamento de Psicología, Universidad Católica del Maule \\ ${ }^{3}$ Facultad de Ciencias de la Educación, Universidad Católica del Maule
}

\section{Resumen}

El artículo tiene como propósito analizar las representaciones acerca de la responsabilidad personal docente en la motivación escolar. Participaron del estudio profesores en formación y en ejercicio de Educación primaria $(n=773)$ de la Región de la Araucanía, Chile. A partir de un análisis de cluster se identificaron cuatro perfiles distintos, en donde esta responsabilidad se asume como propia o compartida y donde la motivación escolar es representada como algo continuo o como un momento acotado de la clase. Los resultados muestran que habría un desplazamiento de la responsabilidad docente hacia la familia, en torno a la motivación escolar, cuando se ejerce efectivamente la profesión. Además, los profesores en ejercicio tenderían concebir la motivación escolar como un momento acotado y de responsabilidad compartida; mientras que los futuros profesores participan significativamente más del perfil en donde la motivación es un proceso continuo y de responsabilidad del docente. Dado que el profesor es el agente que con mejores herramientas debería ser capaz de dar sentido y motivar el aprendizaje escolar, urge generar estrategias para que esta responsabilidad sea asumida de manera contundente por el propio profesor y como un proceso, más allá de un momento específico de la clase.

\section{Palabras clave}

Responsabilidad Docente; Motivación escolar; profesores; estudiantes de pedagogía.

\section{Contacto:}

La correspondencia de este artículo dirigirla a Jorge Valenzuela, jvalenzuela@ucm.cl, Facultad de Ciencias de la Educación, Universidad Católica del Maule. Av. San Miguel 3605, Talca, Chile. Esta investigación ha sido financiada por la Comisión Nacional de Investigación en Ciencia y Tecnología (Conicyt-Chile) a través de los Proyectos Fondecyt Regular 1150533 y 1181159 


\title{
Teaching personal responsibility and school motivation
}

\begin{abstract}
This article analyzes self-reports of teacher perceptions regarding personal responsibility in school motivation. Participants were teachers-in-training and those currently working in Primary Education $(n=773)$ in the Araucanía Region, Chile. Four different profiles were identified from cluster analysis, where responsibility was seen as personal, or shared; and where school motivation was represented as continuous, or discrete. Results show that respondents tend to place responsibility for school motivation within the family for the most effective exercise of the profession. For practicing teachers, school motivation tended to be conceived of as discrete moments, of shared responsibility; while future teachers were significantly more likely to see motivation as a continuous process, of personal (teacher) responsibility. Given that teachers have the educational tools and opportunity to most effectively motivate school learning, it is urgent to generate strategies where responsibility is robustly assumed by teachers themselves; and as a continuous process beyond a specific moment in the classroom.
\end{abstract}

\section{Keywords}

Teacher responsibility; School Motivation; Teachers; Pre-service Teachers

\section{Introducción}

La responsabilidad profesional de los docentes ha sido abordada tradicionalmente a partir de una lógica de rendición de cuentas (accountability). Desde esta perspectiva, la Escuela y los educadores son responsables de los resultados académicos obtenidos por los estudiantes, por ejemplo, en el desempeño en evaluaciones estandarizadas (Biesta, 2015; Biscarra, Giaconi, \& Assaél, 2015; Feng, Figlio, \& Sass, 2018).

Esta forma de entender la responsabilidad profesional que le cabe al profesor enfatiza la importancia de una "responsabilidad asignada" de manera externa, por sobre el "sentimiento personal de responsabilidad" frente a su labor profesional (Lauermann, 2014; Lauermann \& Karabenick, 2013).

En la última década, las políticas de estandarización y responsabilización por desempeño han configurado en Chile una nueva "gramática educativa" (Falabella \& De la Vega, 2016) caracterizada por la definición de estándares de desempeño y de calidad, la aplicación de sistemas de evaluación del aprendizaje (nacionales e internacionales) a lo que se agrega la publicación y sistema de clasificación de los resultados obtenidos por cada centro escolar. Esta política le asigna a las escuelas, en general, y al profesor, en particular, la responsabilidad de mejorar resultados educativos y evitar el abandono escolar (Falabella \& De la Vega, 2016; Matteucci, Guglielmi, \& Lauermann, 2017). Esta forma de responsabilización configura la experiencia profesional de los profesores, quienes son interpelados por una política pública definida por dispositivos de control tales como estándares, desempeños, incentivos, sanciones, entre otros (Assaél, Albornoz, \& Caro, 2018; Oliva \& Gascón, 2016). 
Esta responsabilidad externa o asignada sobre la labor docente no es neutra. La evidencia internacional disponible nos permite inferir que la lógica de rendición de cuentas (accountability) tiene efectos negativos en las prácticas pedagógicas de los docentes y en la calidad del proceso educativo, donde el acto de aprender se reduce a una mera respuestaestímulo del aprendiz, en función de lo que el educador pueda predecir y controlar (Ehren \& Hatch, 2013; Manzi, Bogolasky, Gutiérrez, Grau, \& Volante, 2014).

En Chile se ha cuestionado el rol de las políticas de rendición de cuentas en la transformación de la cultura escolar, debido a que el énfasis en la calidad educativa, la gestión escolar y las buenas prácticas pedagógicas es reemplazado por el predominio de prácticas que promueven la reducción y estandarización del contenido curricular (Ascorra, Carrasco, López, \& Morales, 2019; Núñez-Valdés, Medina-Pérez, \& González-Campos, 2019; Quaresma \& Orellana, 2016). Esta reducción y estandarización ha provocado que los estudiantes sean adiestrados en función de las pruebas que deben completar (teaching to the test) (Falabella, 2016; Resnick \& Schantz, 2017) y el empleo de "soluciones" poco éticas para obtener mejores resultados (ej. excluir de las evaluaciones a los estudiantes con bajo rendimiento o con NEE) (Superintendencia de Educación).

Estas prácticas provocarían que los docentes desvaloricen su labor profesional. Su sentido de identidad profesional es percibido como trastocado: los docentes no saben si educar o enseñar para los exámenes (Darling-Hammond, Wilhoit, \& Pittenger, 2014). Así, la política pública y la sociedad le exigen al profesor responsabilidad por los resultados que obtienen los estudiantes en pruebas estandarizadas más que por una educación integral de los estudiantes.

Asumir esta política no está libre de tensión. Algunos profesores la asumen irreflexivamente, mientras que en otros se genera una tensión entre la vocación, sus opciones pedagógicas/autonomía profesional, y estas demandas del sistema (Fuentealba Jara \& Imbarack Dagach, 2014) p. 259).

\section{Responsabilidad personal docente}

Responsabilizarse por las labores encomendadas por la Política Pública Educativa no es lo mismo que asumir una responsabilidad personal frente a las diversas necesidades que, tanto la sociedad como la práctica profesional, demandan al ejercicio docente. Así, asumir una responsabilidad personal implicaría asumir como propios ciertos desafíos profesionales, dinámica a la que la literatura ha denominado responsabilidad personal docente (teacher's sense of responsibility) (Daniels, Poth, \& Goegan, 2018; Daniels, Radil, \& Goegan, 2017; Lauermann \& Karabenick, 2013; Matteucci et al., 2017).

En este contexto, la literatura ha definido el constructo de responsabilidad personal docente como "un sentimiento de obligación y compromiso interno" en pos de resultados educativos (Lauermann \& Karabenick, 2013, p. 13). Esta dinámica puede ser de dos tipos, por una parte, esta responsabilidad influye o genera acciones concretas o dispositivos para lograr los objetivos deseados y por otra, este sentimiento de responsabilidad genera el evitamiento de situaciones que pueden ser un freno o un impedimento a que estos objetivos educativos se concreten. Así, este sentimiento de responsabilidad docente se caracteriza por su carácter intrínseco y estaría orientado a producir resultados educativos. Esta caracterización deja fuera las demandas profesionales externas, que si bien pueden ser cumplidas, no son entendidas o internalizadas por los profesores como algo inherente a la misión (vocacional) de ser profesor.

La responsabilidad personal del profesor respecto a su labor profesional es clave tanto en contextos formales, por ejemplo en la forma en la cual realiza y/o asume las obligaciones 
profesionales demandadas por la Escuela (Mineduc, 2012) como informales, por ejemplo, sentirse responsable para ofrecer ayuda más allá de lo estrictamente pedagógico (Lauermann \& Karabenick, 2013).

Por otra parte, esta definición nos pone alerta frente a otros constructos que podrían confundirse con la responsabilidad personal docente.

\section{Responsabilidad personal v/s Accountability}

El sistema de rendición de cuentas asume que los educadores son responsables del logro de sus estudiantes, pero no asume si ellos se sienten personalmente responsables por su labor. Estos dos procesos no necesariamente van de la mano. De ser así, implicaría comprender que un educador que se siente personalmente responsable de sus labores profesionales posee un alto sentido de autodeterminación, está dispuesto a esforzarse sin la necesidad de control externo, actúa como su propio juez y acepta voluntariamente una responsabilidad que va más allá de sus obligaciones formales (Daniels, Radil, \& Wagner, 2016). En definitiva, mientras que la rendición de cuentas requiere monitoreo y control externo, la responsabilidad personal implica motivación interna y autorregulación (Lauermann, Karabenick, Carpenter, \& Kuusinen, 2017).

\section{Responsabilidad v/s Autoeficacia}

Otra confusión habitual es concebir la responsabilidad como sinónimo de autoeficacia (Maddux, 2016; Vancouver, Alicke, \& Halper, 2018). Los primeros estudios sobre responsabilización (Lee \& Smith, 1996, 1997) tendieron a operacionalizar la responsabilidad personal del profesor a partir de formulaciones y criterios diseñados originalmente para medir la autoeficacia (Lauermann \& Karabenick, 2013). Ítems como "Me siento confiado en lograr que mis estudiantes..." son los primeros intentos por operacionalizar la responsabilidad personal del docente.

Con todo, las creencias de los profesores en torno a su capacidad para producir resultados (oriented-approach) no implica necesariamente que sientan responsabilidad por hacerlo (Lauermann et al., 2017). Por ello es importante no confundir ambos constructos y conceptualizar la responsabilidad personal como aquel sentimiento de obligación y compromiso de los docentes frente a su tarea profesional.

\section{Las creencias de los docentes sobre responsabilidad}

El sistema de creencias de los educadores configuran su sentido de responsabilidad personal (Fives \& Buehl, 2012). Este sistema es uno de carácter multifacético que incluye concepciones, actitudes, creencia, o teorías subjetivas (Pajares, 1992; Preckel, Baudson, Krolak-Schwerdt, \& Glock, 2015; Richardson, 1996). A través de esas creencias, los educadores aprenden a interpretar la información que nutre sus procesos de enseñanza y alimenta sus prácticas educativas (Pajares, 1992; Rowan \& Townend, 2016). De esta manera, explicitar cuál es la responsabilidad personal que los educadores le asignan a su labor profesional permitiría mejorar su proceso de enseñanza y sus prácticas de instrucción (Lemon \& Garvis, 2016; Matheis, Kronborg, Schmitt, \& Preckel, 2017; Matteucci et al., 2017; Subban \& Mahlo, 2017).

Así, en la medida que los docentes sostienen creencias orientadas a que ciertas labores son propias de su identidad docente, estarán más disponibles para asumirlas y evidenciar un compromiso comportamental con ellas. En sentido inverso, en la medida que el profesor entiende que ciertas labores específicas no le corresponden, aun cuando le sean exigidas por el medio o el sistema educativo formal, tendería a des-responsabilizarse de esas tareas traspasando la responsabilidad a otros actores. 
Así, mientras el proceso de aproximación motivacional se condice con una mayor reponsabilización, el proceso de evitamiento "desresponsabiliza", lo que se manifestaría en el traspaso de responsabilidad a otros actores.

\section{Responsabilidad docente y motivación escolar}

Dentro de las responsabilidades que se le asignan a la función docente, el Marco para la Buena Enseñanza (MINEDUC, 2008), destaca como responsabilidades profesionales la preparación de la enseñanza, la enseñanza para el aprendizaje de todos los estudiantes y la creación de un ambiente propicio para el aprendizaje; así como la actualización profesional, la relación con la familia y el trabajo en equipo.

De estas tareas o funciones asignadas al profesor, hay una que nos interesa en particular: la creación de un ambiente propicio para el aprendizaje donde el factor motivacional es fundamental. Suscitar la motivación escolar, es decir, mejorar el deseo de aprender de nuestros estudiantes, es una labor que tanto la cultura pedagógica como la política pública destacan como una labor propia de los profesores. Pero ies asumida como una responsabilidad profesional por los docentes?

Evidencia reciente muestra que la responsabilidad de la motivación no es algo que los profesores entiendan como una responsabilidad exclusiva de ellos. Junto con identificar otros actores relevantes hay una conceptualización diferenciada sobre qué significa motivar, cuándo es labor del docente y cuándo es labor de la familia (Precht, Valenzuela, Muñoz, \& Sepúlveda, 2016).

\section{Las creencias sobre la motivación}

Un aspecto contextual de la responsabilidad docente es la caracterización concreta de la actividad motivacional. Una aproximación a ello es si la motivación es considerada como un proceso continuo o como un periodo acotada de clase. En el primer caso la motivación se desarrolla a lo largo la clase incentivando el deseo de aprender o realizando una serie de actividades para llamar la atención de los estudiantes a fin de facilitar el proceso de enseñanza (Precht et al., 2016). Una segunda manera de ver la motivación escolar es como un momento concreto y acotado de la hora lectiva: el 'momento' de la motivación al inicio de la clase. En este segundo caso, la motivación está limitada a una decena o quincena de minutos al inicio de la sesión (Martinic \& Villalta, 2015).

\section{El presente estudio}

El presente estudio tiene por objetivo explorar la atribución de responsabilidad de la motivación escolar en profesores en formación y en ejercicio. Nuestras hipótesis postulan diferencias entre profesores en formación y profesores en servicio, a saber:

1) Los profesores en formación conciben que a la motivación hay que dedicarle un tiempo significativamente más extendido que los profesores en ejercicio,

2) Los profesores en formación asignarán significativamente más responsabilidad a la labor propia (del profesor) que los docentes en ejercicio,

3) los docentes en ejercicio asignarían una responsabilidad significativamente mayor a la familia, respecto de la motivación escolar de sus hijos, que sus pares en formación. 


\section{Método}

El presente estudio es parte de un proyecto más amplio (Fondecyt Regular XXXX) que tuvo como finalidad analizar las representaciones sobre la motivación escolar en profesores en ejercicio y en formación. En este contexto, se analizaron las preguntas respecto a responsabilidad en la motivación escolar y el tiempo que se le debe destinar en la clase.

\section{Participantes}

La muestra de este estudio estuvo constituida por un total de 773 profesores de Educación Básica (primaria) en formación ( $n=501)$ y en ejercicio $(n=272)$.

El grupo de futuros profesores estuvo constituido mayoritariamente por mujeres (70.5\%) porcentaje levemente es inferior a la media nacional de profesores de primaria. Su edad promedio era de 23.7 años ( $d s=5.02$ ), y corresponden a estudiantes de pedagogía de universidades privadas y públicas de la región de la Araucanía, Chile, y cursaban la última etapa de su formación profesional en la universidad ( $4^{\circ}$ y $5^{\circ}$ año). En Chile, la formación de profesores se realiza exclusivamente en las universidades y tiene una duración que varía entre 4.5 y 5 años (Contreras-Sanzana \& Villalobos-Clavería, 2010).

Cabe consignar que todos los estudiantes que participaron en la muestra, así como la gran mayoría de los estudiantes de pedagogía chilenos, son formados a través de un modelo pedagógico que considera prácticas tempranas de inmersión en la Escuela (prácticum). Estas prácticas permiten el acercamiento del futuro profesor a la realidad escolar. Esta experiencia permite apropiarse de manera paulatina del rol docente (Gorichon, Ruffinelli, Pardo, \& Cisternas, 2015).

Por su parte, el grupo de docentes en ejercicio ( $75.7 \%$ mujeres) estuvo constituido por docentes cuya media de años de experiencia alcanzaba a 16,06 años (ds=12.74). Se trata de profesores en ejercicio que se desempeñan en escuelas privadas y públicas en diversas ciudades de la región de la Araucanía.

\section{Instrumentos}

Para dar cuenta de la atribución de responsabilidad de diversos actores respecto de la motivación escolar, se les pidió a todos los participantes que respondieran a la pregunta: "En su opinión, ¿a quién le corresponde hacerse cargo de la motivación escolar?". Al mismo tiempo, se pidió a los participantes que asignaran porcentaje de responsabilidad a cuatro agentes potenciales: a) el profesor b) la familia c) el propio estudiante y, finalmente, se agregó la alternativa de d) Otro. La pregunta estuvo diseñada de tal manera que siempre fuese patente la necesidad de que la suma de porcentajes fuese 100. De hecho, no hubo sumas distintas a $100 \%$.

Para indagar sobre la representación de la motivación en su dimensión temporal, es decir, cuán extenso debe ser el tiempo destinado a esta dimensión de la clase, se les preguntó a la los participantes "¿Cuánto tiempo de la clase se debe destinar a motivar a los estudiantes?" Se explicitó además que se debía considerar una clase estándar de 90 min.

\section{Procedimientos}

La toma de datos cumplió con todos los estándares éticos para este tipo de investigación. Entre otros, la aprobación del comité de ética de la institución patrocinante del proyecto y la firma de consentimientos informados de todos los participantes. En todos los casos, la toma de datos fue realizada por uno de los investigadores del equipo y contó con la colaboración de ayudantes. 
Como se señaló anteriormente, este estudio se circunscribe a un aspecto puntual dentro de una investigación más amplia sobre las representaciones de la motivación escolar. En este contexto los participantes contestaron un cuestionario que incluían las preguntas que analizamos en este trabajo. El tiempo de aplicación de la batería completa no excedió los 30 minutos. Como agradecimiento por la participación se sorteó entre todos los participantes 10 tablets.

\section{Resultados}

Tanto para estudiantes de pedagogía como profesores en ejercicio la principal atribución de responsabilidad de la motivación escolar recae en el profesor y luego, en proporciones variables, a la familia y al propio estudiante. En ambos grupos este agente encabeza una estructura de 4 niveles (subconjuntos homogéneos) en donde las puntuaciones de los distintos agentes se diferencian significativamente entre ellos $(p<.001)$.

Junto con lo anterior, cabe constatar que en el caso de los estudiantes de pedagogía, el segundo agente con más responsabilidad es el propio estudiante, mientras que para los profesores en ejercicio lo es la Familia y en ambos grupos, la categoría 'otros' ocupa un muy marginal cuarto lugar.

Tabla 1.

Descriptivos responsabilidad en la motivación escolar por actor (en \%)

\begin{tabular}{|c|c|c|c|c|}
\hline \multirow[t]{2}{*}{ Responsable } & \multicolumn{2}{|c|}{ Estudiantes de pedagogía } & \multicolumn{2}{|c|}{ Profesor en ejercicio } \\
\hline & Media & $\begin{array}{l}\text { Desviación. } \\
\text { Estándar }\end{array}$ & Media & $\begin{array}{c}\text { Desviación. } \\
\text { Estándar }\end{array}$ \\
\hline Profesor & 44.68 & 17.59 & 41.47 & 16.44 \\
\hline Familia & 24.00 & 10.66 & 29.78 & 12.76 \\
\hline Estudiante & 27.28 & 13.24 & 25.77 & 12.39 \\
\hline Otro & 3.99 & 7.42 & 3.13 & 6.13 \\
\hline
\end{tabular}

Para contrastar diferencias eventuales entre los grupos en la responsabilidad asignada a cada agente (profesor, familia, estudiante) se realizó una ANOVA, la cual mostró diferencias significativas entre ambos grupos tanto en la atribución de responsabilidad al profesor como a la familia. En ambos casos hay homocedasticidad en la varianza (Levene $(1,765)=2.49$; $p=.12$ y Levene $_{(1,765)}=1,40 ; p=.24$ ) respectivamente. En el primer caso, los estudiantes de pedagogía asignan significativamente más responsabilidad al profesor que los profesores en ejercicio $\left(F_{(1,765)}=6,08 ; p=.01\right)$. En sentido inverso, son los profesores en ejercicio los que asignan significativamente más responsabilidad en la familia que los estudiantes de pedagogía $\left(F_{(1,765)}=44,54 ; p<.01\right)$. Por su parte al comparar los porcentajes responsabilidad atribuidos al estudiante y la categoría 'otros', no se evidencian diferencias significativas entre los grupos ( $p>.10)$. 


\section{Atribución de responsabilidad y tiempo destinado a la motivación escolar}

Como complemento a la hipótesis de que la atribución de responsabilidad en profesores en formación y en ejercicio sería diferente, se decidió incorporar en el análisis la representación de los profesores sobre el tiempo que se debe asignar a la motivación en la sala de clases. Para ello se realizó un análisis de cluster no jerárquico (K-means).

Los resultados de este análisis permiten identificar cuatro perfiles significativamente distintos entre ellos (ver Tabla 2).

Tabla 2

Descriptivas por clusters

\begin{tabular}{lcccc}
\hline \multirow{2}{*}{ Perfil (Cluster) } & 1 & 2 & 3 & 4 \\
\cline { 2 - 4 } & \multicolumn{1}{c}{$\begin{array}{c}\text { Motivación continua } \\
\text { Responsabilidad } \\
\text { docente }\end{array}$} & $\begin{array}{c}\text { Responsabilidad } \\
\text { compartida }\end{array}$ & $\begin{array}{c}\text { Motivación como momento acotado } \\
\text { Responsilidad } \\
\text { docente }\end{array}$ & $\begin{array}{c}\text { Responsabilidad } \\
\text { compartida }\end{array}$ \\
\hline Tiempo $^{\text {a }}$ & 89,1 & 89,46 & 15,47 & 16,06 \\
Responsabilidad Profesor $^{\mathrm{b}}$ & 60,13 & 31,57 & 39,21 & 30,96 \\
Responsabilidad Familia $^{\mathrm{b}}$ & 18,91 & 29,87 & 19,77 & 31,31 \\
Responsabilidad Estudiante $^{\mathrm{b}}$ & 19,23 & 32,12 & 19,65 & 32,51 \\
\hline Participación en \% $^{10,69 \%}$ & $11,10 \%$ & $33,56 \%$ & $44,65 \%$ \\
\hline
\end{tabular}

a Cuánto tiempo de la clase se debe destinar a motivar (sobre 90 min)

${ }^{\mathrm{b}}$ Responsabilidad atribuida distintos agentes en \%

Perfil 1: Motivación continua - Responsabilidad docente

Se caracteriza por concebir la motivación escolar como una actividad transversal a la clase, es decir, donde la motivación no tiene un momento determinado dentro de la clase sino que la involucra en su totalidad. En este contexto, este primer perfil se caracteriza porque la responsabilidad de motivar a los estudiantes recae fundamentalmente en el docente (60.13\%) y que si bien una parte de la responsabilidad es atribuida a la familia (18.91\%) o al propio estudiante (19.23\%) dichas responsabilidades son menores frente a la responsabilidad del docente.

Perfil 2: Motivación continua- Responsabilidad compartida-(no exclusiva del profesor)

Un segundo perfil o cluster, comparte con el primero la concepción de que la motivación escolar es una actividad permanente durante la clase, pero se diferencia del primero en que la responsabilidad de la motivación escolar se distribuye en porcentajes similares entre el profesor (31.57\%), la familia (29.87\%) y el propio estudiante (32.12\%). Mirado desde otro punto de vista, la responsabilidad de motivar a los estudiantes recae mayoritariamente en otros actores distintos al profesor.

Perfil 3: Motivación como momento acotado - Responsabilidad docente

El tercer perfil, a diferencia de los anteriores concibe que la "motivación escolar" es un momento acotado de la clase, lo que se refleja en la asignación de un tiempo acotado a la 
motivación escolar, del orden de una quincena de minutos. En este contexto el responsable fundamental de la motivación escolar es el profesor (59.21\%) y donde los otros actores (familia y el propio estudiante) tienen una baja responsabilidad en la motivación escolar (19.77\% y $19.65 \%$ respectivamente).

Perfil 4: Motivación como momento acotado Responsabilidad compartida (no exclusiva del profesor)

Finalmente, el cuarto perfil que emerge de este análisis de cluster también considera la motivación como un momento acotado de la clase. Sin embargo, y a diferencia del perfil anterior, en este grupo (que es el más numeroso), la responsabilidad de la motivación escolar es compartida en partes iguales entre el docente, la familia y el propio estudiante (30.96\%; 31.31\% y $32.51 \%$ respectivamente).

Esta clasificación explica una parte importante de la variabilidad que se observa en las variables que dieron origen a esta. Muestra de ello es el resultado de la MANOVA que consideró como factor el cluster y como variable dependiente el tiempo asignado a la motivación escolar y la responsabilidad atribuida al profesor, la familia y el propio estudiante. Este análisis da cuenta que la explicación de la varianza alcanza un $73.1 \%$ ( $\lambda_{\text {wilks }}=$ .019, $\left.F_{(12,2202)}=5260.97 ; p<.001 ; \eta_{\mathrm{p}}^{2}=.731\right)$.

\section{Participación en cluster por tipo de profesor}

Finalmente, se analizó si la participación en estos cuatro clusters difiere entre los profesores en formación (estudiantes de pedagogía) y los que ya están en ejercicio.

Los resultados muestran diferencias significativas en la participación de ambos grupos en los diferentes clusters $\left(\chi_{(1,3)}^{2}=29.75 ; p<.001\right)$.

De manera más específica, se observa que los estudiantes de pedagogía están sobrerepresentados y el grupo de profesores en ejercicio está subrepresentado en el cluster 1, es decir, en el perfil en donde la motivación es una actividad permanente y donde la motivación escolar es fundamentalmente una responsabilidad del docente. Así, mientras que lo esperado era que hubiesen del orden de 50 estudiantes y 28 profesores en este perfil, los resultados muestran que los futuros profesores fueron 71 y sólo hubo 8 docentes en este grupo. En síntesis, este perfil es significativamente más presente en los profesores en formación que en los que ya están en ejercicio.

Un segundo perfil en donde la participación de estos dos tipos de profesores difiere significativamente es el cluster 4 , donde la motivación escolar corresponde a un momento acotado de la clase y donde la responsabilidad de la motivación escolar se distribuye de manera homogénea entre docentes, familia y el propio estudiante. En este perfil, los profesores en ejercicio tienen una participación significativamente más alta de lo esperado y a su vez, los estudiantes de pedagogía, más bajo de lo que se proyectaba. 
Tabla3

Tabla de contingencia Participación en los distintos clusters por tipo de profesor

\begin{tabular}{|c|c|c|c|c|}
\hline \multirow[b]{2}{*}{ Perfil } & & \multicolumn{2}{|c|}{ Tipo de profesor } & \multirow[b]{2}{*}{ Total } \\
\hline & & $\begin{array}{l}\text { Estudiantes } \\
\text { Pedagogía }\end{array}$ & $\begin{array}{l}\text { Profesor en } \\
\text { ejercicio }\end{array}$ & \\
\hline \multirow{4}{*}{$\begin{array}{l}\text { Motivación continua } \\
\text { Resp. docente }\end{array}$} & Recuento & $71_{a}$ & $8 \mathrm{~b}$ & 79 \\
\hline & Frecuencia esperada & 50,9 & 28,1 & 79,0 \\
\hline & \% dentro de Cluster & $89,9 \%$ & $10,1 \%$ & $100,0 \%$ \\
\hline & $\begin{array}{l}\% \text { dentro de Tipo de } \\
\text { Profesor }\end{array}$ & $14,9 \%$ & $3,0 \%$ & $10,7 \%$ \\
\hline \multirow{4}{*}{$\begin{array}{l}\text { Motivación continua } \\
\text { Responsabilidad } \\
\text { compartida - }\end{array}$} & Recuento & $58 a$ & $24 a$ & 82 \\
\hline & Frecuencia esperada & 52,8 & 29,2 & 82,0 \\
\hline & $\%$ dentro de Cluster & $70,7 \%$ & $29,3 \%$ & $100,0 \%$ \\
\hline & $\begin{array}{l}\% \text { dentro de Tipo de } \\
\text { Profesor }\end{array}$ & $12,2 \%$ & $9,1 \%$ & $11,1 \%$ \\
\hline & Recuento & $155 a$ & $93 a$ & 248 \\
\hline \multirow{3}{*}{$\begin{array}{l}\text { Motivación como momento } \\
\text { acotado } \\
\text { Responsabilidad docente- }\end{array}$} & Frecuencia esperada & 159,7 & 88,3 & 248,0 \\
\hline & $\%$ dentro de Cluster & $62,5 \%$ & $37,5 \%$ & $100,0 \%$ \\
\hline & $\begin{array}{l}\% \text { dentro de Tipo de } \\
\text { Profesor }\end{array}$ & $32,6 \%$ & $35,4 \%$ & $33,6 \%$ \\
\hline \multirow{4}{*}{$\begin{array}{l}\text { Motivación como momento } \\
\text { acotado } \\
\text { Responsabilidad. } \\
\text { compartida }\end{array}$} & Recuento & $192 a$ & $138_{b}$ & 330 \\
\hline & Frecuencia esperada & 212,6 & 117,4 & 330,0 \\
\hline & $\%$ dentro de Cluster & $58,2 \%$ & $41,8 \%$ & $100,0 \%$ \\
\hline & $\begin{array}{l}\% \text { dentro de Tipo de } \\
\text { Profesor }\end{array}$ & $40,3 \%$ & $52,5 \%$ & $44,7 \%$ \\
\hline
\end{tabular}

**Cada letra de subíndice indica un subconjunto de Tipo de Profesor categorías cuyas proporciones de columna no difieren significativamente entre sí en el nivel $p<.05$.

\section{Discusión}

La evidencia recogida en este estudio indica que habría un desplazamiento de la responsabilidad docente concerniente la motivación escolar desde el profesor hacia la familia. Es decir, en la etapa de formación la responsabilidad de motivar a los estudiantes que se le asigna al profesor es significativamente mayor que en aquellos en ejercicio de la profesión. Esta baja en la atribución al profesor como responsable de la motivación de los estudiantes se ve compensada en los profesores en ejercicio con un responsablización significativamente mayor que el grupo de estudiantes de pedagogía a la familia como agente motivador.

Pese a que no se trata de un estudio longitudinal que permita controlar en los mismos individuos estas variables, permite avizorar que existe un cambio respecto a cómo se concibe la labor docente respecto a la motivación escolar. Estudios recientes muestran que los profesores conciben como condición necesaria a su labor, el aporte motivacional que realizan las familias. Son ellas las que dan sentido al aprendizaje, mientras que la labor docente se acotaría a atraer la atención de los estudiantes para poder "pasar la materia" (Precht et al., 2016). Esto sería consistente, además, con el hecho de que una gran mayoría de los profesores en ejercicio sean clasificables dentro del cluster 3, el cual concibe la motivación como un espacio acotado en la clase (Martinic \& Villalta, 2015) y con una responsabilidad compartida, donde la mayor parte de la responsabilidad está situada de manera externa al docente (familia, estudiantes, otros) y que una ínfima porción de ellos 
(3\%) se sitúe en el cluster 1 , esto es que concibe la motivación como una actividad permanente durante la clase y cuya responsabilidad recae fundamentalmente en el profesor.

Estos resultado nos alertan frente a un hecho manifiesto: el profesor es el agente que con mejores herramientas debería ser capaz de dar sentido y motivar el aprendizaje escolar (Mineduc, 2012) Trasladar la responsabilidad de dar sentido a los aprendizajes en contexto escolar a otros agentes, como por ejemplo la familia, implica desligarse de una responsabilidad que otros no siempre pueden asumir (e.g. tiempo, formación).

¿Qué hace que esta responsabilidad docente de motivar el aprendizaje disminuya en el ejercicio de la profesión docente? Este estudio no tiene la respuesta, pero deja el desafío para saber por qué estaría pasando esto.

Con todo, los resultados expuestos, sugieren una baja en la responsabilidad personal docente (Lauermann et al., 2017) al restringir, por una parte, la labor motivacional a una espacio acotado de la clase, junto con atribuir una responsabilidad a la familia frente a la cuestión motivacional, más allá de lo que parece razonable. Lo anterior, contribuye a un llamado de alerta en dos direcciones. 1. Mejorar la formación motivacional de los docentes en orden a concebir la motivación centrada en el aprendizaje como una dinámica continua durante la clase, sin reducirla al "momento de la motivación" (Valenzuela, Muñoz, SilvaPeña, Gómez, \& Precht, 2015), y 2, un llamado de atención a que suscitar el interés por aprender y dar sentido al aprendizaje escolar es una tarea que debe concernir fundamentalmente al docente (Viau, 2009, 2013) y que no puede banalizarse, restringiéndola a una dinámica de entretención (Valenzuela, Muñoz, \& Montoya Levinao, 2018).

\section{Referencias}

Ascorra, P., Carrasco, C., López, V., \& Morales, M. (2019). Políticas de Convivencia Escolar en Tiempos de Rendición de Cuentas. education policy analysis archives, 27(31-33), 1-24. doi: http://dx.doi.org/10.14507/epaa.27.3526

Assaél, J., Albornoz, N., \& Caro, M. (2018). Estandarización educativa en Chile: tensiones y consecuencias para el trabajo docente. Educação Unisinos, 22(1), 83-90. doi: http://doi.org/10.4013/edu.2018.221.09

Biesta, G. (2015). What is education for? On good education, teacher judgement, and educational professionalism. European Journal of Education, 50(1), 75-87. doi: https://doi.org/10.1111/ejed.12109

Biscarra, C., Giaconi, C., \& Assaél, J. (2015). El docente en la legislación educacional chilena. Psicoperspectivas, 14(3), 80-92.

Contreras-Sanzana, G., \& Villalobos-Clavería, A. (2010). La formación de profesores en Chile: una mirada a la profesionalización docente. Educación y Educadores,, 13(3), 397-417. doi: http://doi.org/10.5294/edu.2010.13.3.5

Daniels, L. M., Poth, C., \& Goegan, L. D. (2018). Enhancing our understanding of teachers' personal responsibility for student motivation: A mixed method study. Frontiers in Education, 3, 91. doi: http://doi.org/10.3389/feduc.2018.00091

Daniels, L. M., Radil, A., \& Wagner, A. K. (2016). Concordance Between Preservice Teachers' Personal Responsibilities and Intended Instructional Practices. The Journal of Experimental Education, 84(3), 529-553. 
doi:https://doi.org/10.1080/00220973.2015.1054333

Daniels, L. M., Radil, A. I., \& Goegan, L. D. (2017). Combinations of personal responsibility: Differences on pre-service and practicing teachers' efficacy, engagement, classroom goal structures and wellbeing. Frontiers in Psychology, 8, 906. doi: http://doi.org/10.3389/fpsyg.2017.00906

Darling-Hammond, L., Wilhoit, G., \& Pittenger, L. (2014). Accountability for college and career readiness: Developing a new paradigm. Education Policy Analysis Archives/Archivos Analíticos de Políticas Educativas, 22, 1-34. doi: http://dx.doi.org/10.14507/epaa.v22n86.2014

Ehren, M. C., \& Hatch, T. (2013). Responses of schools to accountability systems using multiple measures: The case of New York City elementary schools. Educational Assessment, Evaluation and Accountability, 25(4), 341-373. doi: https://doi.org/10.1007/s11092-013-9175-9

Falabella, A. (2016). ¿ Qué aseguran las políticas de aseguramiento de la calidad?: un estudio de casos en distintos contextos escolares. Estudios pedagógicos (Valdivia), 42(1), 107126. doi: http://dx.doi.org/10.4067/S0718-07052016000100007

Falabella, A., \& De la Vega, L. F. (2016). Políticas de responsabilización por desempeño escolar: Un debate a partir de la literatura internacional y el caso chileno. Estudios pedagógicos (Valdivia), 42(2), 395-413. doi: http://dx.doi.org/10.4067/S071807052016000200023

Feng, L., Figlio, D., \& Sass, T. (2018). School accountability and teacher mobility. Journal of Urban Economics, 103, 1-17. doi: https://doi.org/10.1016/j.jue.2017.11.001

Fives, H., \& Buehl, M. M. (2012). Spring cleaning for the "messy" construct of teachers' beliefs: What are they? Which have been examined? What can they tell us. In K. R. Harris, S. Graham, \& T. Urdan (Eds.), APA educational psychology handbook (Vol. 2, pp. 471-499). Washington DC: American Psychological Association.

Fuentealba Jara, R., \& Imbarack Dagach, P. (2014). Compromiso docente, una interpelación al sentido de la profesionalidad en tiempos de cambio. Estudios pedagógicos (Valdivia), 40(Especial), 257-273. doi: http://dx.doi.org/10.4067/S071807052014000200015

Gorichon, S., Ruffinelli, A., Pardo, A., \& Cisternas, T. (2015). Relaciones entre Formación Inicial e Iniciación profesional de los docentes. Principios y desafíos para la formación práctica Cuadernos de Educación (Vol. 66). Santiago: Universidad Alberto Hurtado.

Lauermann, F. (2014). Teacher responsibility from the teacher's perspective. International Journal of Educational Research, 65, 75-89. doi: https://doi.org/10.1016/j.ijer.2013.09.005

Lauermann, F., Karabenick, S., Carpenter, R., \& Kuusinen, C. (2017). Teacher motivation and professional commitment in the United States: The role of motivations for teaching, teacher self-efficacy and sense of professional responsibility. In $\mathrm{H}$. Watt, P. Richardson, \& K. Smith (Eds.), Global Perspectives on Teacher Motivation (pp. 322348). Cambridge, UK: Cambridge University Press.

Lauermann, F., \& Karabenick, S. A. (2013). The meaning and measure of teachers' sense of responsibility for educational outcomes. Teaching and Teacher Education, 30, 13-26. doi: https://doi.org/10.1016/j.tate.2012.10.001 
Lee, V. E., \& Smith, J. B. (1996). Collective Responsibility for Learning and Its Effects on Gains in Achievement for Early Secondary School Students. American Journal of Education, 104(2), 103-147. doi: http://doi.org/10.1086/444122

Lee, V. E., \& Smith, J. B. (1997). High school size: Which works best and for whom? Educational Evaluation and Policy Analysis, 19(3), 205-227. doi: https://doi.org/10.3102/01623737019003205.

Lemon, N., \& Garvis, S. (2016). Pre-service teacher self-efficacy in digital technology. Teachers and Teaching, 22(3), 387-408. doi: https://doi.org/10.1080/13540602.2015.1058594

Maddux, J. E. (2016). Self-efficacy. In S. Trusz \& P. Bąbel (Eds.), Interpersonal and Intrapersonal Expectancies (pp. 55-60). London: Routledge.

Manzi, J., Bogolasky, F., Gutiérrez, G., Grau, V., \& Volante, P. (2014). Análisis sobre valoraciones, comprensión y uso del SIMCE por parte de directores escolares de establecimientos subvencionados. (Informe Proyecto FONIDE F711269). Santiago: Ministerio de Educación de Chile.

Martinic, S., \& Villalta, M. (2015). La gestión del tiempo en la sala de clases y los rendimientos escolares en escuelas con jornada completa en Chile. Perfiles Educativos, 37(147), 28-49. doi: https://doi.org/10.1016/j.pe.2013.03.001

Matheis, S., Kronborg, L., Schmitt, M., \& Preckel, F. (2017). Threat or challenge? Teacher beliefs about gifted students and their relationship to teacher motivation. Gifted and Talented International, 32(2), 134-160. doi: https://doi.org/10.1080/15332276.2018.1537685

Matteucci, M. C., Guglielmi, D., \& Lauermann, F. (2017). Teachers' sense of responsibility for educational outcomes and its associations with teachers' instructional approaches and professional wellbeing. Social Psychology of Education, 20(2), 275-298. doi: https://doi.org/10.1007/s11218-017-9369-y

MINEDUC. (2008). Marco para la Buena Enseñanza. Santiago: Centro de Perfeccionamiento, Experimentación e Investigaciones Pedagógicas (CPEIP ). Ministerio de Educación, Chile.

Mineduc. (2012). Estándares orientadores para egresados de carreras de pedagogía en educación básica ( $2 a$ ed.). Santiago: Ministerio de Educación.

Núñez-Valdés, K., Medina-Pérez, J. C., \& González-Campos, J. (2019). Impacto de las habilidades de comprensión lectora en el aprendizaje escolar: Un estudio realizado en una comuna de la región metropolitana, Chile. Revista Electrónica Educare, 23(2), 1-22. doi: https://doi.org/10.15359/ree.23-2.2

Oliva, M. A., \& Gascón, F. (2016). Estandarización y racionalidad política neoliberal: bases curriculares de Chile. Cadernos Cedes, 36(100), 301-318. doi: http://doi.org/10.1590/CC0101-32622016171349

Pajares, F. (1992). Teachers' beliefs and educational research: Cleaning up a messy construct. Review of Educational research, 62(3), 307-332. doi: http://doi.org/10.3102/00346543062003307

Precht, A., Valenzuela, J., Muñoz, C., \& Sepúlveda, K. (2016). Familia y motivación escolar: desafíos para la formación inicial docente. Estudios pedagógicos (Valdivia), 42(4), 165-182. doi: http://doi.org/10.4067/S0718-07052016000500010 
Preckel, F., Baudson, T. G., Krolak-Schwerdt, S., \& Glock, S. (2015). Gifted and maladjusted? Implicit attitudes and automatic associations related to gifted children. American Educational Research Journal, 52(6), 1160-1184. doi: https://doi.org/10.3102/0002831215596413

Quaresma, M. L., \& Orellana, V. (2016). El accountability y su impacto en la labor docente: percepciones de los profesores de liceos públicos de alto rendimiento académico en Chile. Currículo sem Fronteiras, 16(2), 316-338.

Resnick, L. B., \& Schantz, F. (2017). Testing, teaching, learning: who is in charge? Assessment in Education: Principles, Policy \& Practice, 24(3), 424-432. doi: http://doi.org/10.1080/0969594X.2017.1336988

Richardson, V. (1996). The role of attitudes and beliefs in learning to teach. In J. Sikula (Ed.), Handbook of research on teacher education (2nd ed., Vol. 2, pp. 102-119). New York: Macmillan.

Rowan, L., \& Townend, G. (2016). Early career teachers' beliefs about their preparedness to teach: Implications for the professional development of teachers working with gifted and twice-exceptional students. Cogent Education, 3(1), 1242458. doi: http://doi.org(10.1080/2331186X.2016.1242458

Subban, P., \& Mahlo, D. (2017). 'My attitude, my responsibility 'Investigating the attitudes and intentions of pre-service teachers toward inclusive education between teacher preparation cohorts in Melbourne and Pretoria. International Journal of Inclusive Education, 21(4), 441-461. doi: https://doi.org/10.1080/13603116.2016.1197322

Superintendencia de Educación. Superintendencia de Educación y Agencia de Calidad: "Ningún estudiante puede ser discriminado durante la aplicación del Simce" [Press release]. Retrieved from https://www.supereduc.cl/prensa/superintendencia-deeducacion-y-agencia-de-calidad-ningun-estudiante-puede-ser-discriminado-durantela-aplicacion-del-simcel

Valenzuela, J., Muñoz, C., \& Montoya Levinao, A. (2018). Estrategias motivacionales efectivas en profesores en formación. Educaçao e Pesquisa, 44, e179652. doi: http://doi.org/10.1590/S1678-4634201844179652

Valenzuela, J., Muñoz, C., Silva-Peña, I., Gómez, V., \& Precht, A. (2015). Motivación escolar: Claves para la formación motivacional de futuros docentes. Estudios pedagógicos (Valdivia), 41(1), 351-361. doi: http://doi.org/10.4067/S0718-07052015000100021

Vancouver, J. B., Alicke, M., \& Halper, L. R. (2018). Self-Efficacy. In D. Lance Ferris, R. E. Johnson, \& C. Sedikides (Eds.), The Self at Work: Fundamental Theory and Research (pp. 15-38). London: Routledge.

Viau, R. (2009). La motivation en contexte scolaire Bruxelles: De Boeck.

Viau, R. (2013). La motivation à apprendre des étudiants mieux comprendre pour mieux agir. [Conference à I'Université Catholique de Lyon]. Retrieved from https://www.youtube.com/watch?v=30h3q-jaigl 